J. Phys. IV France 138 (2006) 105-110

(C) EDP Sciences, Les Ulis

DOI: $10.1051 / j p 4: 2006138013$

\title{
Effet de la pression de mélange sur le rayonnement VUV du xénon dans le cas d'enseignes lumineuses sans mercure excitées en régime d'impulsions électriques
}

\author{
S. Point ${ }^{1}$, E. Robert ${ }^{1}$, S. Dozias ${ }^{1}$, C. Cachoncinlle ${ }^{1}$, R. Viladrosa ${ }^{1}$ \\ et J.-M. Pouvesle ${ }^{1}$ \\ ${ }^{1}$ GREMI-Polytech'Orléans, 14 rue d'Issoudun, BP. 6744, 45067 Orléans Cedex 2, France
}

\begin{abstract}
Résumé. Cette contribution se propose de présenter les derniers développements d'une étude dont l'objectif est d'améliorer l'efficacité énergétique et les performances lumineuses d'enseignes sans mercure excitées en régime impulsionnel. Ces études ont montré que, pour un mélange de néon et de xénon, l'utilisation de pressions relativement élevées permet de favoriser l'émission à $147 \mathrm{~nm}$ du xénon, laquelle est capable d'exciter les phosphores des enseignes. Par ailleurs, on a pu mettre en évidence que l'élévation de pression s'accompagne de la production d'excimères et du développement de bandes d'émissions moléculaires dans l'ultraviolet du vide (VUV).
\end{abstract}

\section{INTRODUCTION}

La directive européenne 2002/96/CE, relative aux Déchets d'Equipements Electriques et Electroniques, stipule que le mercure doit être retiré des lampes à décharges, ce qui inclut les tubes phosphorescents à cathodes froides utilisés dans le domaine des enseignes lumineuses. L'intérêt principal du mercure est ici sa capacité à émettre intensément un rayonnement ultraviolet à $254 \mathrm{~nm}$ de longueur d'onde, ce qui permet d'exciter les phosphores composés de terres rares. Des recherches précédentes menées dans le cadre d'une collaboration entre le GREMI (Orléans), le CPAT ( Toulouse) et la société Aupem Sefli (Gien) ont permis de démontrer l'intérêt des mélanges à bases de gaz rares pour remplacer les dispositifs actuels d'enseignes lumineuses fonctionnant sur des décharges en vapeur de mercure. Il est apparu qu'un mélange binaire néon-xénon est un couple intéressant, compte tenu de la capacité du néon à homogénéiser la décharge, et surtout celle du xénon d'émettre intensément dans le domaine de l'ultraviolet du vide, et en particulier la raie de résonance à $147 \mathrm{~nm}$ (transition $\mathrm{Xe}\left(1 \mathrm{~s}_{4}\right) \rightarrow \mathrm{Xe}\left(1 \mathrm{~s}_{0}\right)$ ), qui permet d'exciter les phosphores. De plus, il a été montré que la durée de vie d'une enseigne à base de gaz rare atteignait celle des dispositifs au mercure. Cependant, les performances de tels mélanges, sous excitation sinusoïdale classique, restaient 5 à 10 fois inférieures à celles des enseignes au mercure, notamment à cause d'excitations successives à partir de Xe $\left(1 \mathrm{~s}_{4}\right)$, qui entraient en concurrence avec la désexcitation radiative. Plus récemment, les recherches se sont donc orientées vers l'étude des performances d'enseignes lumineuses excitées en régime d'impulsions électriques [1] [2], l'objectif étant de créer une excitation suffisamment courte pour limiter les phénomènes d'excitations successives. Ces études ont conduit le GREMI à développer un prototype compact d'alimentation impulsionnelle (dénommé GECOPULSE), capable de fournir des impulsions de quelques kilovolt, dans le domaine microseconde, et à un taux de répétition de plusieurs kilohertz, soit des rapports cycliques (durée d'impulsion multipliée par la fréquence) autour de $10^{-3}$.

Il est apparu par ce biais un gain significatif en termes d'éclairement. En effet, alors qu'une excitation sinusoïdale ne permet pas à l'enseigne de fournir des éclairements supérieurs à 1000 Lux, et ce, quelle que soit la puissance injectée, les performances lumineuses des enseignes sous excitation impulsionnelle augmentent avec la puissance électrique. Ainsi, à $25 \mathrm{~W}$, l'éclairement est 3 fois plus important sous excitation impulsionnelle que sous excitation sinusoïdale. Cette amélioration des performances permet déjà d'envisager l'utilisation d'enseignes sans mercure pour certaines applications commerciales en 
intérieur. On explique cette amélioration par une augmentation du rayonnement à $147 \mathrm{~nm}$ en cours de décharge mais surtout par la production très importante de cette émission à $147 \mathrm{~nm}$ en période de postdécharge. Ainsi, des mesures ont déjà montré que la quantité de lumière VUV produite au cours de la post-décharge, dans les conditions de pression usuelles des enseignes lumineuses, est 2 à 3 fois plus importante que celle produite en période de décharge. Sur ces bases, une nouvelle collaboration s'est mise en place entre le GREMI et Aupem Sefli. Son objectif est d'améliorer encore le dispositif en augmentant ses performances absolues en termes d'éclairement mais surtout son efficacité énergétique, en optimisant les paramètres physiques (pression de mélange, pourcentage des gaz ...) et électriques (largeur temporelle d'impulsion, taux de répétition ...), et en cherchant la meilleure combinaison possible de ces paramètres.

Dans cette étude, nous avons particulièrement travaillé sur l'optimisation des performances énergétiques et lumineuses des enseignes sans mercure sous excitation impulsionnelle en fonction de la pression de mélange sur un large domaine ( 2 ordres de grandeur de pression). On présentera tout d'abord l'influence de la valeur de la pression sur les performances en éclairement, puis le lien qu'il existe entre l'évolution de ces performances et l'évolution de la transition $\mathrm{Xe}\left(1 \mathrm{~s}_{4}\right) \rightarrow \operatorname{Xe}\left(1 \mathrm{~s}_{0}\right)(147 \mathrm{~nm})$. Enfin, on s'intéressera aux modifications du spectre VUV de la décharge avec la pression, dues notamment à la présence de molécules de gaz rares excitées.

\section{DISPOSITIF EXPÉRIMENTAL}

Les travaux ont été réalisés dans un tube expérimental de $14 \mathrm{~mm}$ de diamètre externe et de $0.5 \mathrm{~m}$ de longueur. Un tube de $0.2 \mathrm{~m}$ de long est soudé perpendiculairement au milieu de l'enseigne et permet le remplissage par les gaz et la visée du spectromètre au centre de la colonne positive. Afin d'éliminer les impuretés à l'intérieur de l'enseigne, qui perturbent son fonctionnement et sont en outre néfastes aux observations spectroscopiques, on réalise un dégazage des impuretés, grâce à un bâti de pompage moléculaire double fourni par la société Aupem Sefli. L'enseigne est constamment sous flux de néon (pureté : $99.99 \%$ ) et de xénon (pureté : 99.998\%), ce qui permet de modifier en cours d'expérience la pression totale et les pressions partielles par action sur une vanne et par commande électronique des débitmètres (Brooks $5850 \mathrm{TR}$ ). Les débits utilisés sont de l'ordre de la dizaine $\mathrm{de}^{\mathrm{cm}} / \mathrm{min}$. La pression est contrôlée par une jauge Baratron Alcatel type 623. Une alimentation continue fournit la tension qui est ensuite hachée par GECOPULSE pour former les impulsions. Ces impulsions sont appliquées aux bornes de l'enseigne par l'intermédiaire d'un transformateur élévateur de tension. La tension crête des impulsions est de l'ordre du kilovolt. GECOPULSE délivre des impulsions de largeur variable dans le domaine microseconde dont la fréquence peut être choisie dans une gamme allant de quelques centaines de Hertz à quelques dizaines de kilohertz. La puissance électrique consommée par l'ensemble alimentation/enseigne est maintenue stable. Ceci n'exclut pas d'éventuels écarts avec la puissance directement injectée à l'enseigne, et dont la mesure directe est délicate. Le diagnostic optique est réalisé, par l'intermédiaire d'un spectromètre monochromateur Acton Research Corporation (réseau 1200 traits $/ \mathrm{mm}$, focale : $0.4 \mathrm{~m}$ ), grâce à une Caméra ICCD rapide ( Princeton Instrument), conditionnée sous atmosphère d'azote pour les acquisitions en VUV, ou grâce à un tube photomultiplicateur rapide sensible sur une plage $100 \mathrm{~nm}-300 \mathrm{~nm}$ (Hamamatsu R1080). Les mesures en éclairement (prises sur le phosphore) se font quant à elles grâce à un Luxmètre Minolta.

\section{EXPÉRIENCES ET DISCUSSIONS}

Les mesures réalisées au cours de ces expériences seront présentées en unité arbitraire, pour des raisons de confidentialité. On s'intéressera donc essentiellement à l'évolution des tendances et aux écarts relatifs entre les grandeurs observées. 


\subsection{Performances en éclairement}

Les études sous excitation impulsionnelle ont montré qu'il était possible de produire des décharges luminescentes jusqu'à des valeurs de pression de mélange relativement élevées. Une large gamme de pression a donc pu être explorée. Il est apparu que les caractéristiques de la décharge dépendent de la quantité de xénon dans le mélange (figure 1). Un faible pourcentage de xénon permet d'avoir une décharge diffuse et efficace jusqu'à des valeurs de pression relativement élevées. A contrario, un fort pourcentage de xénon comprime la décharge qui s'éteint rapidement lorsque la pression de mélange s'élève. Un mélange à pression relativement haute avec un faible pourcentage de xénon offre donc les meilleurs éclairements, ce qui en fait un bon candidat pour le développement d'enseignes sans mercure excitées en régime impulsionnel. Il est important de noter qu'une élévation de pression dans un mélange néon xénon sous excitation sinusoïdale entraîne au contraire une diminution des performances lumineuses.

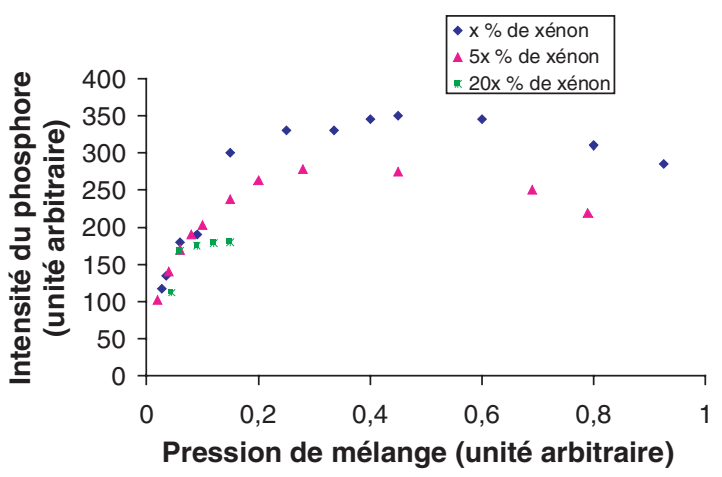

Figure 1. Intensité du phosphore selon la pression de mélange et le pourcentage de xénon sous excitation impulsionnelle.

\subsection{Spectroscopie atomique résolue en temps}

Le spectre VUV de la décharge en néon xénon est essentiellement composé de deux raies spectrales $(129.6 \mathrm{~nm}$ et $147 \mathrm{~nm})$, qui correspondent aux transitions $\mathrm{Xe}\left(1 \mathrm{~s}_{2}\right) \rightarrow \mathrm{Xe}\left(1 \mathrm{~s}_{0}\right)$ et $\mathrm{Xe}\left(1 \mathrm{~s}_{4}\right) \rightarrow \mathrm{Xe}\left(1 \mathrm{~s}_{0}\right)$. La raie à $147 \mathrm{~nm}$ est la plus intense, son rôle est donc déterminant dans l'excitation des phosphores. On a suivi l'évolution de la raie de résonance $\mathrm{Xe}\left(1 \mathrm{~s}_{4}\right) \rightarrow \mathrm{Xe}\left(1 \mathrm{~s}_{0}\right)$ au cours du temps, afin de connaître l'évolution de la répartition de l'énergie lumineuse avec la pression. La figure 2 présente la valeur de la quantité de lumière VUV de la décharge, de la post-décharge, et de l'ensemble, pour la transition considérée, avec une puissance électrique consommée par le dispositif enseigne/alimentation constante, et un pourcentage de xénon faible. On remarque tout d'abord que la plus grande partie du rayonnement VUV est fournie en période de post-décharge, et que l'évolution du rayonnement VUV total est dictée par le rayonnement de post-décharge. On constate que la quantité de lumière VUV est maximum pour une pression de 0.5 unité arbitraire, qui est aussi la pression qui permet d'avoir le maximum d'éclairement d'après les mesures de la figure 1. L'hypothèse retenue pour expliquer l'augmentation de l'émission VUV avec la pression est celle d'une augmentation des processus de désexcitation collisionnelle, qui permettent de peupler le $\mathrm{Xe}\left(1 \mathrm{~s}_{4}\right)$ à partir d'états plus excités (réaction R1, tableau 1 ). Cette augmentation de la densité de neutres excités dans le milieu favorise les collisions ionisantes (réactions R2 et R3). Les ions xénon alors obtenus participent au processus de recombinaison électron/ion qui est à l'origine du repeuplement de $\mathrm{Xe}\left(1 \mathrm{~s}_{4}\right)$ au cours de la post-décharge (réaction R4). Au-delà d'une certaine pression cependant, l'émission à $147 \mathrm{~nm}$ diminue. Des phénomènes de désexcitation collisionnelle (réaction R5) participent à la destruction de $\mathrm{Xe}\left(1 \mathrm{~s}_{4}\right)$. En effet, les valeurs des taux de réactions permettent de penser 


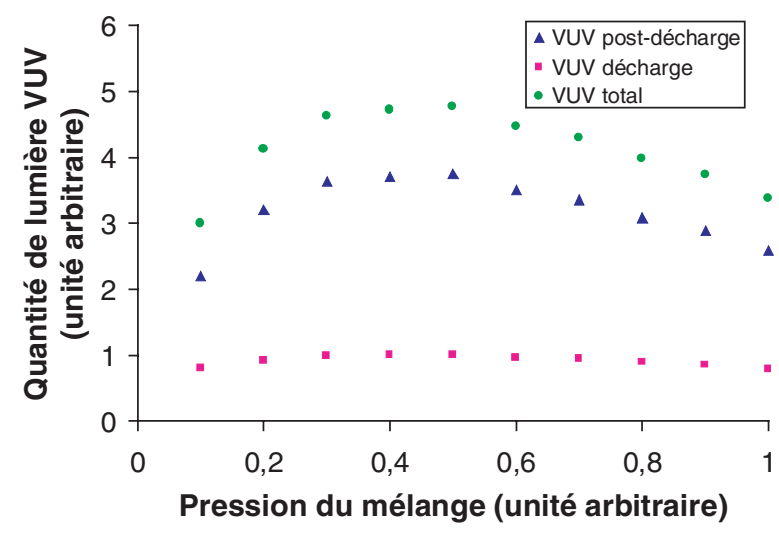

Figure 2. Quantité de lumière VUV selon la période (décharge ou post-décharge) et la pression de mélange.

Tableau 1. Réactions pouvant être impliquées dans la production ou la désexcitation du niveau $\mathrm{Xe}\left(1 \mathrm{~s}_{4}\right)$ à l'origine de l'émission à $147 \mathrm{~nm}$.

\begin{tabular}{|c|l|c|c|}
\hline Réaction & Equation de la réaction & Coefficient de réaction & Référence \\
\hline $\mathrm{R} 1$ & $\mathrm{Xe}^{* *}+\mathrm{Ne} \rightarrow \mathrm{Xe}^{*}+\mathrm{Ne}$ & $12.10^{-9} \mathrm{~cm}^{3} \mathrm{~s}^{-1}$ & {$[3]$} \\
\hline $\mathrm{R} 2$ & $\mathrm{Xe}^{*}+\mathrm{Xe}^{*} \rightarrow \mathrm{Xe}^{*}+\mathrm{Xe}+\mathrm{e}$ & $5.10^{-10} \mathrm{~cm}^{3} \mathrm{~s}^{-1}$ & {$[4]$} \\
\hline $\mathrm{R} 3$ & $\mathrm{Xe}^{-1} \mathrm{Ne}^{*} \rightarrow \mathrm{Xe}^{*}+\mathrm{Ne}+\mathrm{e}$ & $7.10^{-11} \mathrm{~cm}^{3} \mathrm{~s}^{-1}$ & {$[5]$} \\
\hline $\mathrm{R} 4$ & $\mathrm{Xe}^{*}+2 \mathrm{e} \rightarrow \mathrm{Xe}^{*}+\mathrm{e}$ & $\mathrm{Te}^{-4.5 *} 2,8.10^{-27} \mathrm{~cm}^{6} \mathrm{~s}-1$ & {$[6]$} \\
\hline $\mathrm{R} 5$ & $\mathrm{Ne}+\mathrm{Xe}^{*} \rightarrow \mathrm{Ne}+\mathrm{Xe}$ & $10^{-11} \mathrm{~cm}^{3} \mathrm{~s}-1$ & {$[3]$} \\
\hline
\end{tabular}

que, à la pression atteinte lors du maximum d'émission à $147 \mathrm{~nm}$, la vitesse de destruction du résonant par désexcitation collisionnelle est du même ordre de grandeur que la vitesse de désexcitation radiative. Par ailleurs, on observe, pour les plus fortes pressions de la gamme et malgré un pourcentage de xénon assez bas, un début de contraction de la décharge, ce qui n'est pas favorable à l'excitation des phosphores.

Ainsi, l'utilisation d'une excitation impulsionnelle permet de faire fonctionner des décharges dans une large gamme de pression. On a pu mettre en évidence l'existence d'une pression optimale correspondant à la fois au maximum d'émission VUV et au maximum d'éclairement.

\subsection{Spectre d'émission moléculaire}

L'élévation de pression dans une décharge de gaz rares peut entraîner l'apparition de molécules diatomiques excitées, les excimères. Nous avons mené des expériences afin de vérifier si nos conditions expérimentales permettaient l'obtention de telles molécules, et plus particulièrement le xénon moléculaire excité, $\mathrm{Xe}_{2}{ }^{*}$, susceptible d'émettre une quantité non négligeable de rayonnement VUV. Une large fenêtre du spectre VUV a donc été acquise par la caméra, préalablement conditionnée sous une atmosphère d'azote. Le mélange contenait un faible pourcentage de xénon. On a pu observer (figure 3) l'existence du premier continu moléculaire à partir de $147 \mathrm{~nm}$ environ et l'interruption de cette émission vers $210 \mathrm{~nm}$ de longueur d'onde, ce qui correspond au point tournant gauche du puit de potentiel de la molécule (On notera que l'émission à 147 nm est trop intense pour être observée intégralement à la même échelle). L'émission du premier continu augmente en même temps que la pression de mélange. L'élévation de pression favorise en effet la création de dimères excités, notamment le $\mathrm{Xe}_{2} *$. Le flux VUV (obtenu en sommant le flux élémentaire de chaque longueur d'onde de l'émission considérée) fourni par l'émission de premier continu du xénon (entre 150 et $210 \mathrm{~nm}$ ) représente moins de $10 \%$ de 


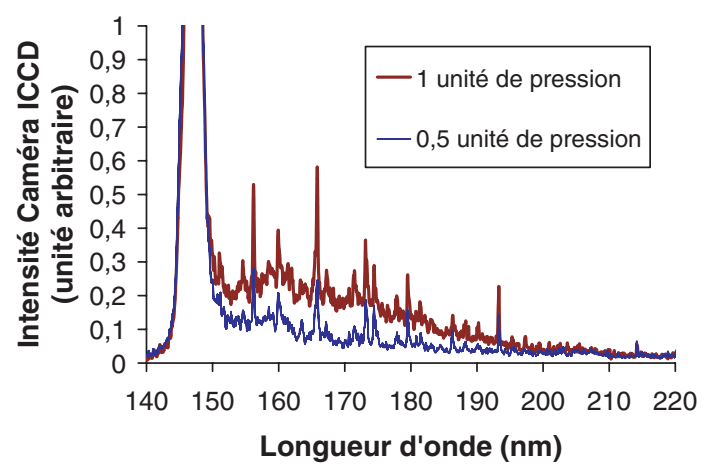

Figure 3. Emission de premier continu du xénon.

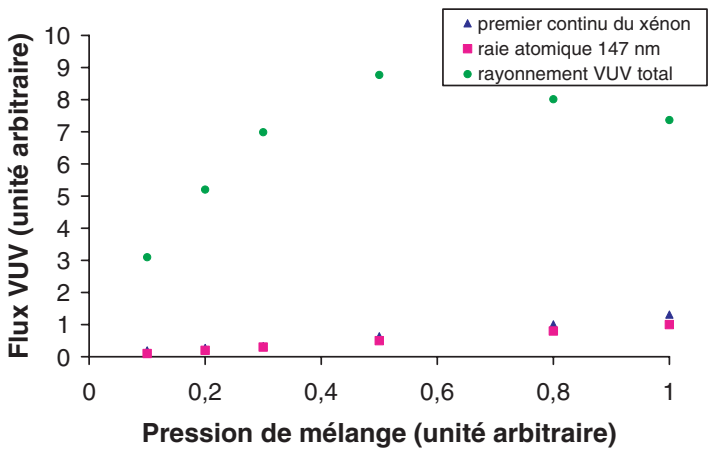

Figure 4. Comparaison des différentes émissions VUV (atomique et moléculaire).

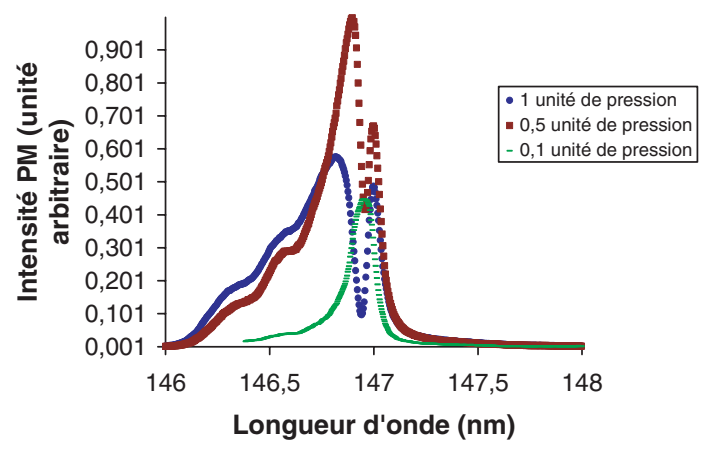

Figure 5. Aile bleue de la raie $147 \mathrm{~nm}$.

l'émission VUV totale à 0.5 unité de pression, mais atteint près de $20 \%$ de l'émission VUV totale aux plus fortes pressions de la gamme (figure 4). Il faut noter que le flux VUV atomique (147 nm) n'a pas sur ce graphique été corrigé de l'absorption due à l'épaisseur de gaz dans le tube soudé à l'enseigne. En outre, on a pu identifier la présence de $\mathrm{NeXe}^{*}$ responsable de l'apparition d'une aile bleue à la raie de résonance à $147 \mathrm{~nm}$ (figure 5). La structure en bande observée, conséquence de transitions libre-libre et libre-liée au sein de la molécule NeXe*, s'accorde bien avec les résultats de A.Morozov et al [7]. Il est difficile de distinguer, dans cette aile bleue, la stricte contribution lumineuse de la queue de l'émission atomique à $147 \mathrm{~nm}$ (due à l'élargissement résonant de la raie) et celle de l'émission moléculaire. Cependant, la contribution de cette aile bleue avoisine celle du premier continu.

Comme on pouvait s'y attendre, l'élévation de la pression de mélange, sous excitation impulsionnelle, permet la formation de molécules de gaz rares excitées, connues pour émettre en larges bandes dans le domaine VUV. Au plus fortes pressions utilisées, ces bandes d'émissions moléculaires représentent un pourcentage non négligeable du rayonnement VUV total participant à l'excitation du phosphore.

\section{CONCLUSION}

On a pu mettre en évidence que l'élévation de pression dans un mélange Ne/Xe, associée à un faible pourcentage de xénon, permettait d'améliorer les performances lumineuses des enseignes. En effet, l'élévation des densités favorise le peuplement de $\mathrm{Xe}\left(1 \mathrm{~s}_{4}\right)$, dont la relaxation produit le rayonnement 
à $147 \mathrm{~nm}$. On a également montré que cette émission à $147 \mathrm{~nm}$ atteignait un maximum pour une valeur de pression de mélange donnée. Au-delà de celle-ci, des désexcitations collisionnelles détruisent $\mathrm{Xe}\left(1 \mathrm{~s}_{4}\right)$, ce qui a pour conséquence de diminuer l'émission à $147 \mathrm{~nm}$ et l'excitation des phosphores.

L'augmentation de la pression provoque également le développement d'émissions VUV continues, traduisant l'existence d'excimères. Ces émissions sont capables d'exciter les phosphores mais semblent, dans la gamme de pression observée, ne pas pouvoir compenser suffisamment la diminution de la raie de résonance à $147 \mathrm{~nm}$.

L'utilisation de pression de mélange assez élevée avec un faible pourcentage de xénon apparaît donc comme une voie prometteuse pour le développement d'enseignes lumineuses sans mercure. De nouveaux travaux sur l'influence de la nature du gaz tampon devront être réalisés sous excitation impulsionnelle. Actuellement, les travaux portent sur l'influence de divers paramètres de l'impulsion électrique sur la cinétique réactionnelle et les performances lumineuses.

\section{Remerciements}

Ce travail est financé par l'ADEME et le Conseil Régional Centre.

\section{Références}

[1] E. Robert and al, Light sources 2004, p. 485-486.

[2] E. Robert et al, J. Phys. IV France 127 (2005) p. 39-42.

[3] R. Cooper and al, Phys. Rev. A, 50 (1994) p. 4812.

[4] S. Rauf and M.J. Kushner, J. Appl. Phys. 85 (1999) p. 3460.

[5] B. Min and al, J. Vac. Technol. A, 18 (2000) p. 349.

[6] L.M. Biberman, V.S. Vorobjev and I.T. Yakubov, 1987, Kinetics of Noneequilibrium LowTemperature Plasma (New York : Plenum).

[7] A. Morozov et al, J. Phys. D : Appl. Phys. 36 (2003) 1126-1134. 